\title{
Etalonnages absolus dans le domaine X-UV
}

\author{
P. Troussel
}

CEA Centre d'Etudes de Limeil-Valenton, 94195 Villeneuve-Saint-Georges cedex, France

\section{Résumé :}

L'étalonnage des détecteurs de rayonnement $\mathrm{X}$-mou utilisés pour le diagnostic des plasmas créés par laser, trouve dans le rayonnement synchrotron du LURE un outil précieux. Nous montrons comment caractériser l'énergie de sortie d'une ligne de lumière avec un bolomètre et une photodiode, la structure spatiale avec une caméra $\mathrm{CCD}$ et sa structure temporelle avec une caméra à balayage de fente. Nous présentons à titre d'exemples des résultats d'étalonnage absolu de détecteurs.

\section{Introduction :}

La nécessité d'étalonner en valeur absolue les chaînes de mesure utilisées pour étudier les plasmas créés par laser a conduit le CEA/DAM à instrumenter des lignes de lumière du synchrotron de SUPER-ACO du LURE.

Le CEL-V a développé un montage expérimental portable constitué d'un goniomètre travaillant dans les deux polarisations et d'un système pour la caractérisation des détecteurs $X$ et d'optiques X [1]. Ce système donne des informations sur la structure spatiale (CCD), spectrale (bolomètres, photodiodes, channeltron...), et temporelle (caméras à balayage de fente) du faisceau de sortie. Nous l'avons utilisé par campagne de mesures derrière des monochromateurs à réseaux dans le domaine 50-1000 eV. Dans le domaine $1 \mathrm{keV}-10 \mathrm{keV}$ nous avons disposé nos détecteurs derrière le dispositif de la ligne SB3 du centre d'études du CEBIII de Bruyères-le-Châtel equipée d'un monochromateur à deux cristaux indépendants et d'un goniomètre [ 2]. Nous présentons quelques résultats d'étalonnage absolu servant à connaître la réponse de nos détecteurs X.

\section{Caractérisation spectrale :}

\section{II.1. Mesures du nombre de photons :}

Le rayonnement synchrotron est un outil précieux pour caractériser spectralement les détecteurs de RX. La première étape consiste à mesurer le nombre de photons à la sortie de la ligne de lumière en fonction de l'énergie sélectionnée par un monochromateur a l'aide d'un bolomètre ou d'une photodiode.

Un bolomètre, d'une surface de $1 \mathrm{~cm} 2$, développé initialement pour mesurer le rayonnement V.U.V. et $\mathrm{X}$ mou émis par des tokomaks a été adapté pour mesurer 
$\mathrm{l}^{\dagger}$ énergie émise par un plasma crée par l'interaction laser matière. Ce bolomètre est constitué d'une feuille de capton $(7.5 \mu \mathrm{m})$ avec une couche d'or $(5 \mu \mathrm{m})$ absorbant le rayonnement sur une face et une résistance d'or $(5 \mathrm{kOhm})$ sur l'autre face. Il fonctionne comme un Wattmètre [3]. Il est capable de mesurer des densités de puissances supérieures à $10^{-7} \mathrm{~W} / \mathrm{cm}^{2}$. Sa sensibilité spectrale est constante dans la gamme $5 \mathrm{eV}$ - $5 \mathrm{keV}$. La figure 1 montre la puissance spectrale mesurée en sortie de la ligne SU7 de Super-Aco derrière un wiggler : l'énergie des photons est sélectionnée par deux réseaux dans la gamme $150-600 \mathrm{eV}$ et $300-1000 \mathrm{eV}$. Elle est comparée au flux environ 10 fois plus faible, issue de la ligne SA23 placée à la sortie d'un aimant de déviation dans la gamme $30-200 \mathrm{eV}$. On remarque qu'à $284 \mathrm{eV}$ peu de rayonnement atteint le bolomètre à cause d' une couche mince de Carbone déposée sur au moins un élément optique de la ligne SU7. Les mesures derrière l'aimant entre 30 et $200 \mathrm{eV}$ sont moins précises, à cause du flux plus faible, et on est proche du seuil de sensibilité du bolomètre. Les mesures ont été effectuées avec des fentes du monochromateur assez ouvertes, ce qui correspond à une bande passante d'environ $1 \mathrm{eV}$.

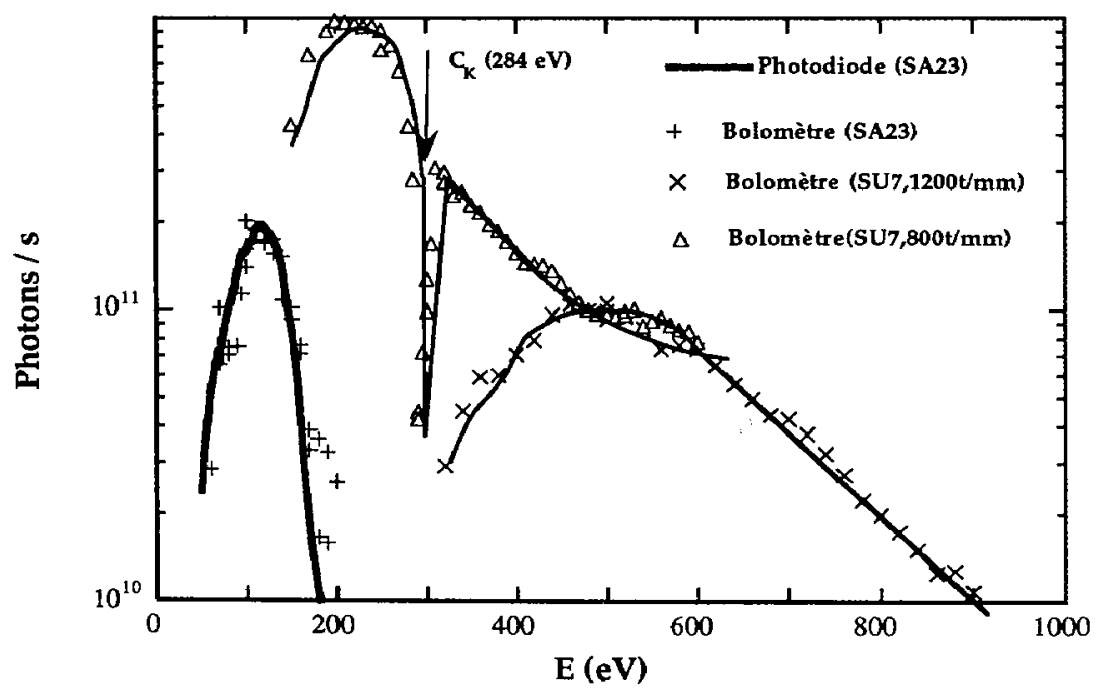

Figure 1 : nombre de photons ramené à un courant synchrotron de $100 \mathrm{~mA}$ mesuré derrière les réseaux de 800 traits $/ \mathrm{mm}$ et 1200 traits $/ \mathrm{mm}$ de la ligne SU7 mesuré avec le bolomètre et derrière la ligne $S A 23$ mesuré avec une photodiode et un bolomètre.

Si l'on veut faire des mesures avec des fentes plus fermées ( bandes passantes inférieures à l'eV), on peut utiliser une cellule semi-conductrice plus sensible. Depuis quelques années, ce type de détecteur a été développé et est utilisé comme détecteur étalon avec une incertitude de l'ordre de $5 \%$. Nous avons testé une photodiode de silicium type UDT XUV 100. Pour le calcul de la réponse spectrale nous utilisons les paramètres déterminés expérimentalement par une méthode d"autoétalonnage" [4] et par comparaison à un bolomètre $[5,6]$ : épaisseur de la zone morte td de $\mathrm{SiO}_{2}(75 \AA)$, région de charge d'espace ts $(10 \mu \mathrm{m})$, longueur de diffusion $(200 \mu \mathrm{m})$.

Le rendement théorique $\mathrm{R}(\mathrm{E})$ exprimé en électron/photon est donné par : 


$$
R(E)=(h v / w) K(E)=(h v / w) e^{\left(-\mu_{d}(h v) t_{d}\right)}\left[1-\frac{e^{\left(-\mu_{s}(h v) t_{s}\right)}}{\mu_{s}(h v) L+1}\right]
$$

où $\mathrm{K}(\mathrm{hv})$ désigne le rendement intrinsèque en électron représenté sur la figure 2 , w représente l'énergie moyenne pour la production d'une paire électron trou dans le silicium $(3.7 \mathrm{eV}), \mu \mathrm{s}$ et $\mu \mathrm{d}$ (E) les coefficients d'absorption respectivement du silicium et de l'oxyde. Les flux de photons ont été calculés à partir du photocourant reçu par la photodiode et de son R. Q. semi-empirique R(E).

Sur la figure 1, la comparaison des flux de photons mesurés avec la photodiode sur la ligne SA23 entre 50 et $200 \mathrm{eV}$ aux mesures bolométriques précédentes est bonne : le bon accord des résultats nous a suggéré par la suite de l'utiliser comme étalon secondaire entre $5 \mathrm{eV}$ et $10 \mathrm{keV}$, en particulier sur la ligne SB3 au dessus du keV.

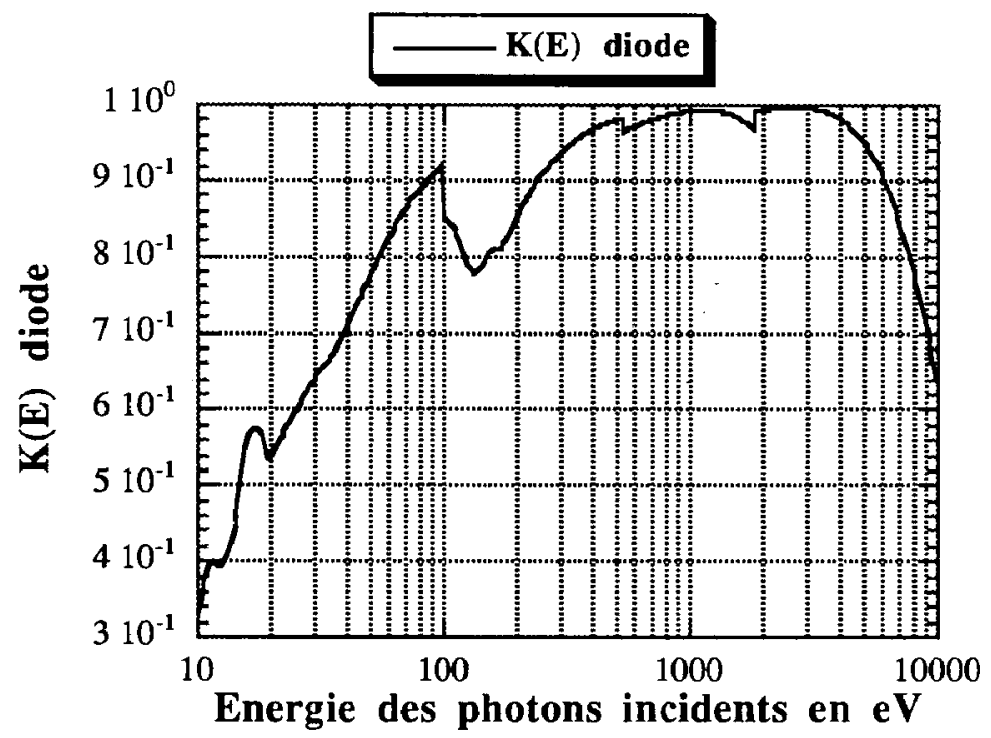

Figure 2 : Rendement intrinsèque limitant la quantité d'électrons générés . Les sauts correspondent aux bords d'absorption dus au fait que les profondeurs de pénétration des photons varie de manière significative par rapport à la profondeur de la zone de charge d'espace $t_{s}$.

Sur la figure 3 nous donnons les flux mesurés en sortie du monochromateur dans les cas où la sélectivité spectrale est réalisée avec deux cristaux de Béryl (figure $3 a)$ ou deux cristaux d'InSb (figure $3 \mathrm{~b}$ ) avec des erreurs du \%. Un filtre de $25 \mu \mathrm{m}$ de Béryllium élimine la lumière visible du rayonnement synchrotron. La fente de collimation, pour des raisons de seuil de sensibilité, est très ouverte $(5 \mathrm{~mm})$. Ces valeurs sont comparées à celles calculées par le logiciel "X-ray vision" développé par T. Moreno. Ce logiciel prend en compte les tables de flux de Super - Aco [7] et le calcul des réflectivités intégrées des deux cristaux d'après le modèle de Hirsch et Ramachandram. Les valeurs de flux calculés sont en moyenne environ 10 fois supérieures aux valeurs expérimentales dans le cas du Béryl et 3 pour I'InSb. Cela vient principalement du fait que les réflectivités intégrées théoriques des cristaux sont nettement plus fortes que les réflectivités expérimentales car on considère des mosaïques de cristaux parfaitement régulières. D'autre part ce logiciel calcule le produit de réflectivités intégrées des deux cristaux en 
considérant une étendue géométrique du faisceau constante quelque soit l'énergie du photon. En fait l'angle d'attaque sur le deuxième cristal change avec l'énergie pour respecter la loi de Bragg, et il peut arriver qu'a haute énergie il y ait une limitation géométrique du faisceau par le deuxième cristal .

Les profils de la figure 3a présente des discontinuités dues aux bords d'absorption de deux éléments Al $(1560 \mathrm{eV})$ et $\mathrm{Si}(1839 \mathrm{eV})$ qui composent le cristal de Béryl. Les différences de niveaux entre les profils de courbes expérimentale et théorique dans le cas du Béryl entre 1000 et $1600 \mathrm{eV}$ sont dus à la présence d' harmoniques des cristaux de Béryl mais aussi à la qualité des cristaux (voir images fig. 6). Le filtre passe-bas en énergie du dispositif permettant d'éliminer ces harmoniques ne peut être utilisé, car la perte de flux qu'il occasionne ne serait pas compatible avec le seuil de sensibilité de la photodiode $\left(10^{6} \mathrm{ph} / \mathrm{s}\right)$.

Les mesures absolues de photons entre 2 et $6 \mathrm{keV}$ avec les cristaux d' InSb sont plus précises à cause du nombre plus important de photons mais aussi parce que ces cristaux ne présentent pas d'harmoniques. L'incertitude de mesure est de l'ordre de $3 \%$.

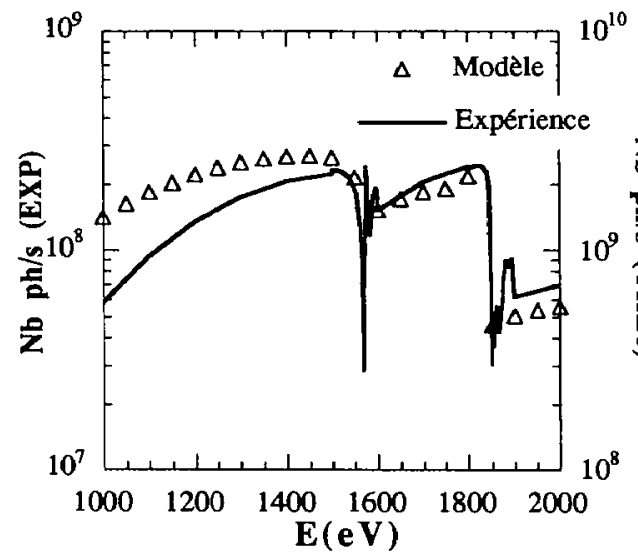

(a)

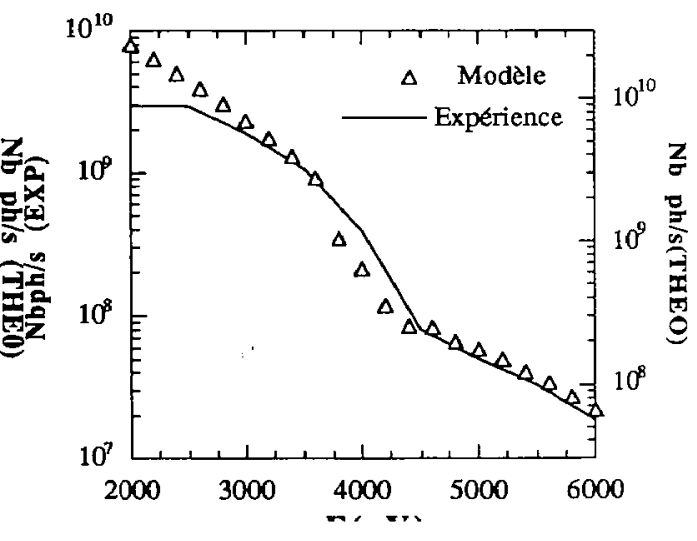

(b)

Figure 3 : Flux de photons théoriques et expérimentaux à la sortie du monochromateur de la ligne SB3 avec deux cristaux de Béryl (a) et d'InSb (b).

\section{2. Applications :}

La connaissance du spectre de sortie a permis de déterminer la réponse spectrale de composants comme des photocathodes, utilisées en transmission dans les caméras à balayage de fente. Elles sont constituées d'une matrice de polymère $(\mathrm{C} 8 \mathrm{H8}) \mathrm{n}$ de faible épaisseur ( 1000 à $2000 \AA$ Å) sur laquelle est déposée une couche conductrice puis éventuellement une couche électro-émissive.

Le R.Q. à une énergie E sélectionnée est obtenue en faisant le rapport du courant d'électrons émis par la photocathode et de la mesure de flux donné par la photodiode. Ainsi par exemple des photocathodes en Aluminium sur $\mathrm{CH}$ et en lodure de Césium sur $\mathrm{Al}$ sur support $\mathrm{CH}$ ont été testées ( figures $4 \mathrm{a}$ a $4 \mathrm{~d}$ ). 

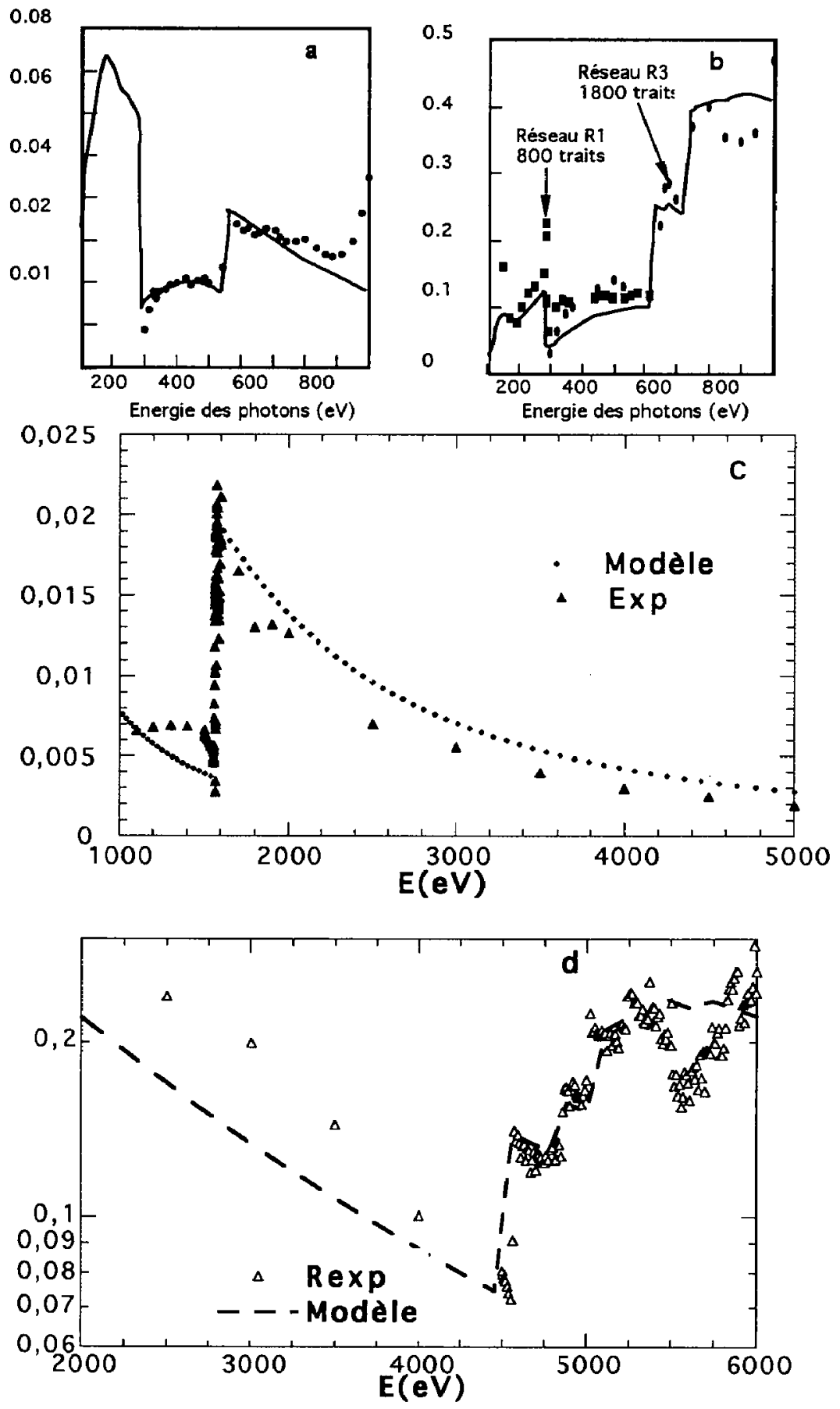

Figure 4 : rendements de photocathodes :

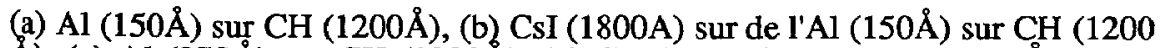

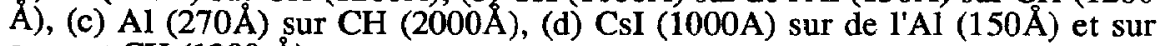
support CH (1200 $\AA$ ). 
Les enregistrements font apparaître l'effet des structures, notamment les bords d'absorption du Carbone à $284 \mathrm{eV}$, de l'Iode (LI $5188 \mathrm{eV}$, LII $4852 \mathrm{eV}$, LIII 4557eV, MI $620 \mathrm{eV}$ ), du Césium (LI $5713 \mathrm{eV}$, LII $5360 \mathrm{eV}$, LIII $5012 \mathrm{eV}$, MI $726 \mathrm{eV}$ ) et de l'Aluminium (K $1560 \mathrm{eV})$.

La comparaison des mesures a été faite à l'aide du modèle de photo-émission décrit par Henke [8] : il a montré que le R.Q. théorique à une énergie de photons E, peut s'écrire :

\section{$R$ (électron/photon) $=\mathrm{C}(\mathrm{E} \mu(\mathrm{E})) \exp (-\mu \mathrm{x})$}

où $x$ est l'épaisseur de la photocathode et $\mu(E)$ le coefficient d'absorption linéique calculé à partir des tables [9]. Nous avons tenu compte dans le modèle d'une épaisseur de $100 \AA$ d'alumine : l'Al se recouvre d'une couche de passivation $\mathrm{d}^{\prime} \mathrm{Al}_{2} \mathrm{O}_{3}$.

On peut voir que ce modèle décrit correctement la dépendance spectrale. Sauf pour le CsI entre 2000 et $4000 \mathrm{eV}$ où les rendements expérimentaux sont plus élevés que ceux prévus par la théorie. L'examen microscopique de la photocathode a mis en évidence une structure en boules, cette structure fait que la photocathode travaille probablement selon deux modes, en transmission et en réflexion. L'incertitude des mesures est de $3 \%$. Notons que le rendement des photocathodes en CsI est d'un ordre de grandeur plus élevé que les photocathodes d'Al.

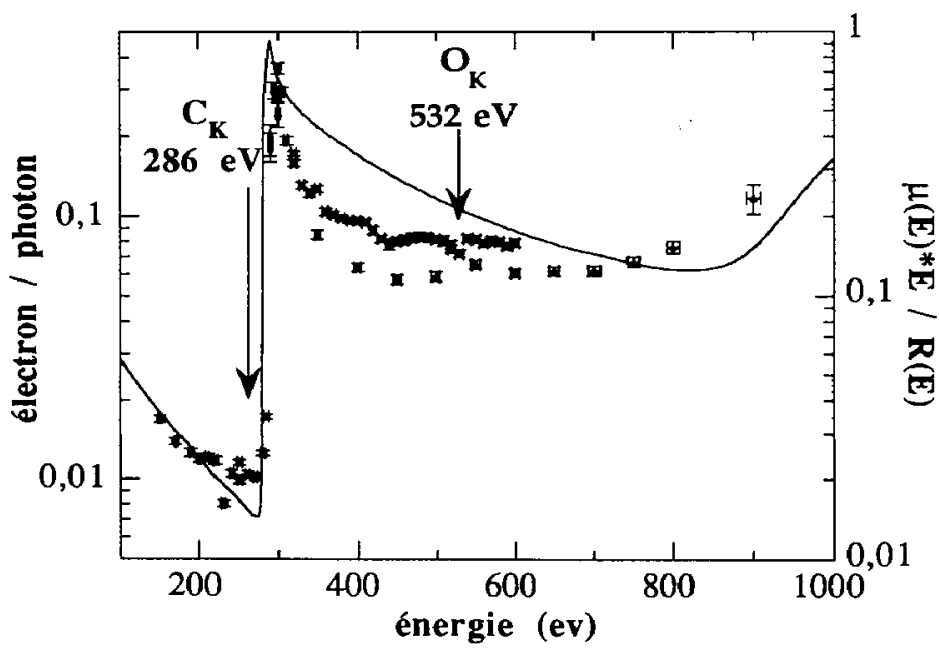

Figure 5 : les points expérimentaux de R.Q. de la feuille "pseudo or" comparés à $\mu(\mathrm{E}) \mathrm{E} / \mathrm{R}$

La ligne de lumière SU7 est alignée en utilisant placée sur le trajet du faisceau une grille d'Or avec une transmission de $80 \%$. Elle est située entre la fente de sortie du monochromateur et le miroir de focalisation. Pour obtenir le rendement de cette photodiode à une énergie donnée (figure 5), le courant mesuré est divisé par la mesure photonique ainsi que par la réflectivité du miroir de sortie. La courbe de rendement calculée d'une grille d'or à l'aide du modèle de Henke a un profil trés différent. Pour restituer la valeur de rendement, nous devons faire l'hypothèse que l'écran et le miroir sont recouverts d'une mince couche de carbone. Cette expérience montre l'intérêt de quantifier les contaminations des 
composants des lignes de lumière synchrotron et le fait que ce type de détecteur n'est pas adapté à la caractérisation spectrale.

\section{Caractérisation spatiale :}

Les images obtenues avec les caméras CCD permettent de localiser la position du faisceau et d'en connaître la répartition spatiale d'énergie [10]. Il est intéressant d'accompagner les mesures spectrales à des caractérisations spatiales pour l'interprétation des résultats. Ainsi les figures (6a) et (6b) présentent deux images typiques du faisceau en sortie de la ligne SB3 respectivement avec les cristaux de Béryls à $2000 \mathrm{eV}$ et avec les InSb à $3000 \mathrm{eV}$ obtenues avec une caméra CCD photométrix [11]. Le faisceau est filtré par une épaisseur de $25 \mu \mathrm{m}$ de Berryllium et avec un temps de pose de $3 \mathrm{~ms}$. Ces deux images montrent les différences de qualité optique des deux types de cristaux (planéité) et expliquent en partie la différence de facteurs que l'on obtenait entre la théorie et l'expérience pour les deux types de cristaux.

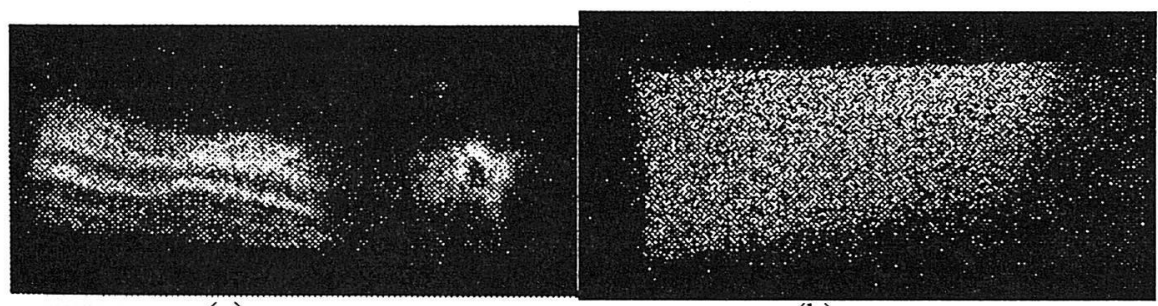

(a)

(b)

Figure 6 : Images du faisceau synchrotron à $2000 \mathrm{eV}$ avec deux cristaux de Béryl (a) et à $3000 \mathrm{eV}$ avec deux cristaux d' InSb (b)

\section{Caractérisation temporelle :}

La structure temporelle du faisceau synchrotron $X$ peut être obtenue à l'aide de caméras à balayage de fente de type TSN506P ayant des tubes P550 dans le domaine des RX mous et P505 dans le domaine UV.

Sur la figure 7 le profil densimétrique montre deux impulsions enregistrées dans le mode synchrotron de 24 paquets espacées de $9.8 \mathrm{~ns}$ et dont la largeur à mi-hauteur est évaluée à 370 ps.

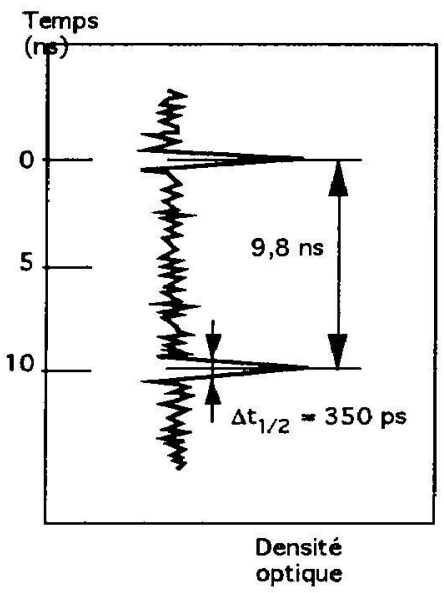

Figure 7 : structure temporelle du faisceau synchrotron enregistrée avec une caméra TSN $550 \mathrm{X}$ à l'ordre zéro du réseau de la ligne SU7 pour une vitesse de balayage de $5 \mathrm{~ns} / \mathrm{cm}$.

La figure 8 représente deux impulsions synchrotrons dans le domaine UV mesurées l'une après l'autre à 1 minute d'écart pour un courant de $96 \mathrm{~mA}$. On observe une modification rapide de la distribution longitudinale du paquet, reliée aux oscillations synchrotron cohérentes et à l'allongement turbulent se produisant 
dans cette gamme de courant. Les modes hexapolaires d'oscillation synchrotron ont ainsi pu être mis en evidence à ce courant. La forme de la distribution est non gaussienne (écart de $20 \%$ ). La distribution longitudinale a pu être mesuré de manière systématique en fonction du courant.

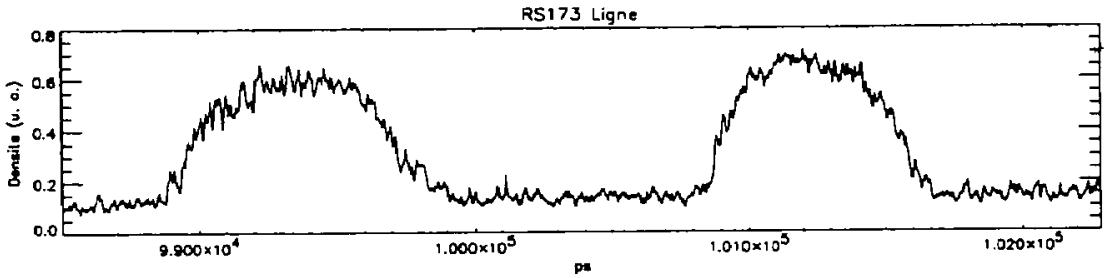

(a)

(b)

Figure 8 : les largeurs temporelles en $1 / \mathrm{e}^{2}$ du faisceau dans le domaine UV valent 230 ps (a) et 186 ps (b).

\section{Conclusion :}

Les progrès réalisés dans la détection X-UV et l'instrumentation [12] permettent de caractériser les faisceaux de sortie des lignes de lumière d'un synchrotron. Les résultats montrent l'intérêt de disposer de lignes de lumière à demeure bien instrumentée afin d'accéder aux flux de photons de sortie, aux propriétés spatiales et temporelles du faisceau synchrotron. L'utilisation du bolomètre ou de photodiode permet de mesurer le nombre de photons a la sortie des lignes de lumière.

\section{Remerciements :}

L'auteur remercie L.Beck, P. Stemmler et G. Soullié pour la mise en oeuvre du monochromateur de la ligne SB3, J. L. Bourgade, J. M. Dalmasso, G. Lidove et C. Reverdin D. Schirmann pour la mise en oeuvre des diagnostics, D. Cuybaynes et G. Rossi pour leurs aides sur les lignes SA23 et SU7 respectivement, M.E.Couprie et D. Gontier pour les mesures de forme temporelle sur le synchrotron.

\section{Références :}

[1] P. Troussel at al SPIE Vol 2011 pp 464 (1993)

[2] Rapport interne CEA (1993) L. Beck, Ph.Stemmler

[3] C. Reverdin, J.L. Bourgade, M. Le Guen, M. Rostaing, P. Troussel, S. Vortisch Inst. Phys. Conf. Ser. No 125: Section 7, 371 (1992)

[4] M. Krumrey and E.Tegeler R. S. I. vol 63 (1992) pp 797

[5] N.Ahr and E.Tegeler N. I. M. A319 (1992) 387

[6] C. Reverdin, P. Troussel, F. LeGuern, J.L. Bourgade, D. Schirmann, J.

M. Dalmasso, D. Gontier, G. Lidove soumis à Laser and particule beams (1994)

[7] P.Thiry, rapport interne LURE 1984/11

[8] B. L.Henke, J.P. Knauer et K. Premaratne J. Appl. Phys. vol 52 pp1509 (1981)

[9] B.L.Henke, P. Lee, T. .Tanaka, R.L. Shimabukuroet B.K.Fujiwaka, At Nucl. Data Tables 271 (1982)

[10] voir papier A. Mens

[11] Rapport d'activité 1993 LULI A. Rousse, G.Geindre, P.Audebert, C. Montheil, F. Fallis, J. C. Gauthier, P.Troussel, D. Mazataud, L.Beck, G. Soullié. [12] Annales UVX 92 D.Schirmann " l'instrumentation dans le domaine X-UV". 